\title{
Intracranial capillary hemangioma misdiagnosed as a meningioma
}

Hemangioma capilar intracraniano assemelhando-se a um meningioma

Carlos B. Dabdoub', Mario Chavez², Jose Luis Ferrufino², Edith Claros ${ }^{3}$, Elisabeth do Nascimento Silveira4, Carlos F. Dabdoub ${ }^{5}$

A 10-year-old boy was admitted to the hospital complaining of headache associated with intermittent vomiting. Non-contrast cranial computed tomography demonstrated a 4.5 × $4.0 \mathrm{~cm}$ well-circumscribed solid lesion in the left parietal region (Figure 1). Magnetic resonance imaging of the brain showed an extra-axial parietal duralbased lesion, with contrast enhancement on T1-weighted images (Figure 2). Additionally, a dural tail sign was evident. Neuroimaging findings were consistent with meningioma but the patient was diagnosed with capillary hemangioma by pathological examination (Figure 3). Therefore, this lesion should be considered in the preoperative diagnosis of extra-axial, avidly enhancing mass lesions with a dural tail sign, especially in children ${ }^{1,2,3}$.
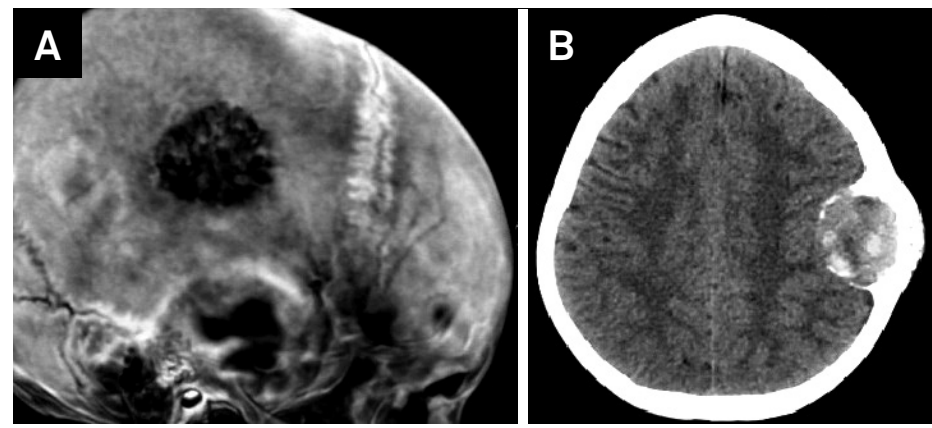

Figure 1. (A) Tridimensional reformatted images from noncontrast CT demonstrate left parietal bone erosion. (B) Noncontrast CT, axial image, disclose and iso / hyperdense left parietal lesion.
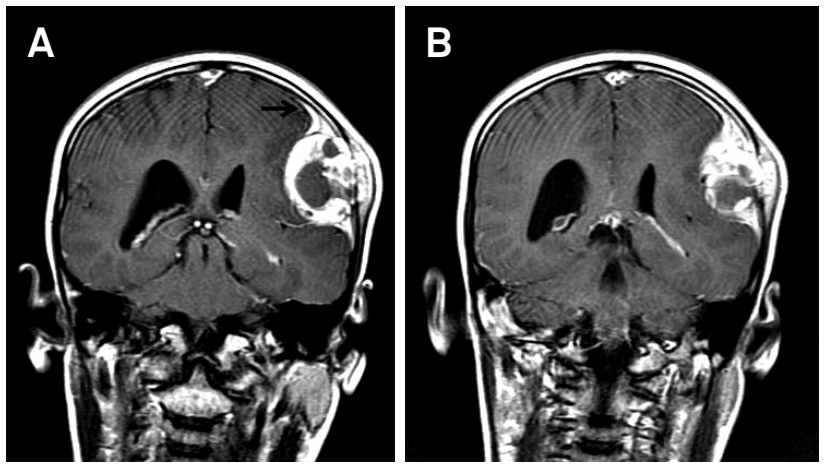

Figure 2. Coronal T1-weighted magnetic resonance image shows a contrast-enhancing extra-axial tumor with a broad dural base in the left parietal region. Note the dural tail sign (arrow).

\footnotetext{
${ }^{1}$ Hospital Municipal do Campo Limpo, Departamento de Neurocirurgia, Sao Paulo SP, Brazil;

${ }^{2}$ Hospital Universitario Japonés, Departamento de Neurocirugía, Santa Cruz de la Sierra, Bolivia;

${ }^{3}$ Centro de Oncología, Departamento de Patología, Santa Cruz de la Sierra, Bolivia;

${ }^{4} U$ niversidade da Cidade de São Paulo, Faculdade de Medicina, Sao Paulo SP, Brazil;

${ }^{5}$ Universidad Franz Tamayo, Facultad de Medicina, Departamento de Neurocirugía, Santa Cruz de la Sierra, Bolivia.

Correspondence: Carlos B. Dabdoub; Hospital Municipal do Campo Limpo, Departamento de Neurocirurgia; Estrada de Itapecerica, 1661; 05835-005 São Paulo SP, Brasil; E-mail: carlosdabdoub@hotmail.com

Conflict of interest: There is no conflict of interest to declare.

Received 09 August 2015; Received in final form 31 October 2015; Accepted 24 November 2015.
} 


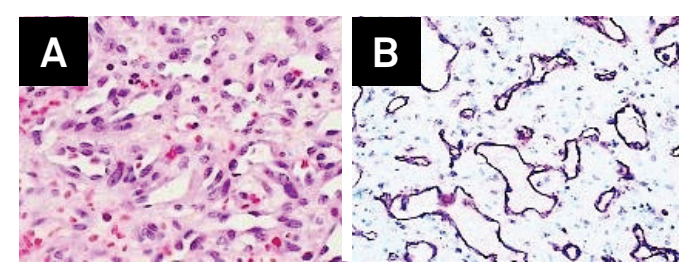

Figure 3. (A) Microscopic findings of the tumor consisted of poorly-defined capillary channels lined by a single layer of endothelial cells without nuclear atypia (Hematoxylin \& eosin; x250). (B) Vascular structures and endothelial lining cells of this tumor show strong expression of vascular markers (CD31immunostaining; x200).

\section{References}

1. Yang G, Li C, Chen X, Liu Y, Han D, Gao X et al. Large capillary hemangioma of the temporal bone with a dural tail sign: A case report. Oncol Lett. 2014;8(1):183-6. doi:10.3892/ol.2014.2143

2. Morace R, Marongiu A, Vangelista T, Galasso V, Colonnese C, Giangaspero F et al. Intracranial capillary hemangioma: a description of four cases. World Neurosurg. 2012;78(1-2):191.E15-21. doi:10.1016/j.wneu.2011.09.017

3. Mirza B, Shi WY, Phadke R, Holton JL, Turner C, Plant GT et al.

Strawberries on the brain - intracranial capillary hemangioma: two case reports and systematic literature review in children and adults. World Neurosurg. 2013;80(6):900.e13-21. doi:10.1016/j.wneu.2012.12.013 